\title{
X-band Band-pass Frequency Selective Surface for Radome Applications
}

\author{
Tariq Rahim ${ }^{\star}$, Jiaodong Xu \\ School of Electronic and Information Engineering, Northwestern Polytechnic University, \\ 710129, Shaanxi, Xi'an, China \\ *Corresponding author, e-mail: rahim372@gmail.com
}

\begin{abstract}
A low profile multi layer miniaturized unit cell frequency selective surface (FSS) with second-order band-pass response is design. The metallic layers in the form of capacitive patches and inductive grids are separated by dielectric substrates. The non-resonant sub-wavelength unit cells with unit cell dimensions and periodicities on the order of $0.15 \lambda$. The overall thickness of approximately $0.03 \lambda$ is designed which is useful at lower frequencies with long wavelengths. The FSS exhibit a stable frequency response to different angles of incidence and polarizations. The analysis and synthesis of the FSS is done using equivalent circuit method and simulated using CST microwave studio at $X$-band.
\end{abstract}

Keywords: FSS, filter, bandpass, radome

Copyright $\odot 2015$ Institute of Advanced Engineering and Science. All rights reserved.

\section{Introduction}

The Frequency selective surfaces (FSSs) have been a great research area for the last few decades. FSS found numerous applications in communication, microwave, millimeter wave and radar systems such as spatial filters [1-3], radar absorbing materials (RAM) [4-6], artificial magnetic conductors (AMC) [7, 8], planar lenses [9, 10], radomes, sub-reflectors, polarizer and reflectarrays antennas [11]. Many methods to reduce the radar cross section (RCS) are to modify the target shape or orientation to deflect the scattered wave away from the detecting radar, converting radio frequency energy to heat by using some RAMs. Most of these techniques have some drawbacks such as the increase of cost and maintenance or they can also affect the global performance of the system. The spatial filter is different from conventional filter is that the spatial filter is more sensitive to angle of incident and the response vary with the incident wave. To achieve higher order band pass response, multiple FSS panels can be cascaded with a quarter wavelength spacing between each panel. This quarter wavelength spacing may be used in some applications which require mechanical strength. But have drawbacks in low-frequency FSSs, as well as conformal FSSs. Moreover, with the introduction of these quarter-wavelength spacers the FSSs become sensitive to angles of incidence. Superstrate dielectric stabilizers are used to remedy the problem but this technique also increase the overall thickness [1]. Dielectric frequency selective surfaces (DFSSs) are introduced which are constructed by inhomogeneous dielectric bars [2]. These structures have the advantage of low absorption loss in contrast to metallic structures, but more difficult to fabricate. A FSS based on substrate integrated waveguide (SIW) technology is studied in [3]. However, they are narrow-band and difficult to construct. FSSs based on microstrip patch antennas have been discussed in [10] and [11], but in all these techniques based on microstrip patch antennas, the size of the structure in the order of half a wavelength which leads to an earlier on setting of grating lobes and will also lose stability of response to different angles of incidence. Therefore, a multi-layered band pass FSSs based on a miniaturized unit cell is proposed at X-band to reduce the RCS of the object and have good pass band response and no harmonics. The elements which constitute the FSS are non-resonant structures which are combined to make a second-order band pass filter. The unit cell size, thickness and the inter element spacing are significantly smaller than the wavelength. This overall small size makes the structure a second order bandpass spatial filter with excellent stable performance with different angles of incident. 
In next section the design procedure and the principles of operation of the proposed FSS are illustrated followed by results and discussion and finally conclusion is drawn accordingly.

\section{Geometry of the Proposed FSS Structure}

The Figure 1 shows the exploded three-dimensional view of the proposed FSS. The FSS contains three different metallic layers isolated by two dielectric substrates Taconic TLX-6 with dielectric constant 2.65. The upper and lower metallic layers provide the capacitance and the central metallic layer form a wire grid and provide inductance to the structure. The thickness of the structure is about twice the thickness of the dielectric substrates $(h)$. Furthermore it is shown that the physical dimensions of $D_{x}$ and $D_{y}$ in the $x$ and $y$ directions, respectively, which are also the same as the period of the structure in the $x$ and $y$ directions. The length of each capacitive metallic patches $D-s$, where, $s$ represents the spacing between the two adjacent capacitive patches. As the structure is symmetrical so the length and width of the unit cell are same and the inductive layer have also length and width $D x=D_{y}=D$, The width of the inductive strip is $w_{1}=w_{2}=w$. The structure is not sensitive to the polarization at normal incidence due to symmetry.

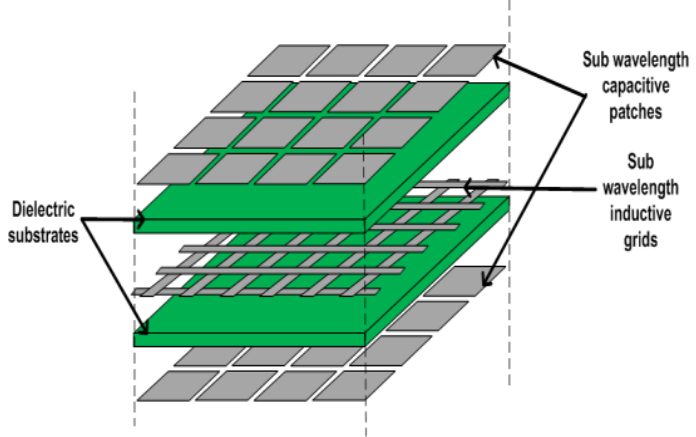

Figure 1. Three dimentional view of the proposed FSS structure

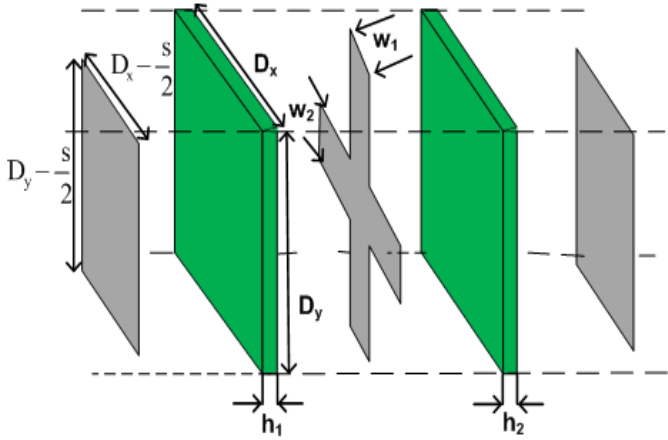

Figure 2. Exploded view of the unit cell FSS with their dimentions

\section{Design Procedure using Equivelent Ciruit Method}

As shown in the Figure 1 the patches are modeled in the equivalent circuit model as $C_{1}$ and $C_{2}$ respectively. The central inductive grids has been model in the equivalent circuit model as $L_{0}$ as depicted in Figure 3 . The two substrates are model as a transmission line with equivalent series inductor and shunt capicitor. The half spaces are also model with semi-infinite transmission model. This equivalent circuit is only valid at normal incidence. The circuit is a second order coupled resonator band pass filter. Figure 3 shows the T-network and their equivalent counterpart m-netowrk. To design a FSS with Butterworth second order response and having values $q_{1}=q_{2}=1.4142, k_{12}=0.70711, r_{1}=r_{2}=1$. Where, $q_{1}$ and $q_{2}$ are the normalized loaded quality factor of the resonators, $k_{12}$, is the normalized coupling coefficient between the two resonators and the normalized source and load impedances, $r_{1}$ and $r_{2}$, which are determined by the desired response category. The fractional bandwidth of the FSS, $\delta$, its center frequency of operation, $f_{0}$, and the response type is Butterworth $2^{\text {nd }}$ order. The relationship between the structure parameters and capacitor and inductor are given [12]:

$$
\begin{aligned}
& C=\varepsilon_{0} \varepsilon_{\text {eff }} \frac{2 D}{\pi} \ln \left(\frac{1}{\sin \frac{\pi s}{2 D}}\right) \\
& L=\mu_{0} \mu_{\text {eff }} \frac{2 D}{\pi} \ln \left(\frac{1}{\sin \frac{\pi w}{2 D}}\right)
\end{aligned}
$$


The values of the equivalent circuit elements shown in Figure 3 are calculate on the basis of Butter worth response parameters using the different mathematical relationships below:

$$
\begin{aligned}
C_{1} & =\frac{q_{1}}{\omega_{0} Z_{0} r_{1} \delta}-\frac{\varepsilon_{0} \varepsilon_{r 1} h_{1}}{2} \\
C_{2} & =\frac{q_{2}}{\omega_{0} Z_{0} r_{2} \delta}-\frac{\varepsilon_{0} \varepsilon_{r 2} h_{2}}{2} \\
L & =\frac{\left(k_{12} \delta\right)^{2}}{1-\left(k_{12} \delta\right)^{2}} \times \sqrt{\frac{r_{1} r_{2}}{q_{1} q_{2}}} \times \frac{z_{0}}{\omega_{0} k_{12}} \\
L_{t 1} & =\frac{k_{12} \delta}{1-\left(k_{12} \delta\right)^{2}} \times\left(1-k_{12} \delta \sqrt{\frac{q_{1} r_{2}}{r_{1} q_{2}}}\right) \times \frac{z_{0} r_{1}}{\omega_{0} q_{1} k_{12}} \\
L_{t 2} & =\frac{k_{12} \delta}{1-\left(k_{12} \delta\right)^{2}} \times\left(1-k_{12} \delta \sqrt{\frac{q_{2} r_{1}}{r_{2} q_{1}}}\right) \times \frac{z_{0} r_{2}}{\omega_{0} q_{2} k_{12}} \\
L_{1} & =L_{t 1}+L_{0}\left(1+\frac{L_{t 1}}{L_{t 2}}\right) \\
L_{2} & =L_{t 2}+L_{0}\left(1+\frac{L_{t 2}}{L_{t 1}}\right) \\
L_{m} & =L_{t 1}+L_{t 2}+L_{0}\left(\frac{L_{t 1} L_{t 2}}{L_{0}}\right)
\end{aligned}
$$

Using the Telegrapher's model for TEM transmission lines, $L_{t}=\mu_{0} \mu_{r} h$ is simply equal to where $\mu_{0}$ is the permeability of free space, $\mu_{r}$ is the relative permeability of the dielectric substrate used and $h$ is the length of the transmission line (equal to the thickness of the dielectric substrate). Using the relationship given in Equation (1) and (2) the required values of the inductor and capacitor is calculated by properly choosing the structural parameters. The gap between the capacitive element should be kept as low as possible keeping the manifuctring constraints into account, the normal slot width using the etching method is $0.15 \mathrm{~mm}$, so we take the value of $s=0.15 \mathrm{~mm}$. The initial value of the inductor strip width $w$ can be also approximated. However, due to close orientation of the metal layers the capacitive patches will affect the value of the inductance of the inductive grids and vice versa. Therefore, the exact dimensions of the capacitive patches and inductive wire grid are optimized using numerical EM simulations [13]. In this manuscript we uses CST microwave studio and unit cell simulation is performed. The Unit Cell Design for FSS template is used in CST which simplifies the design. This template automatically assigns unit-cell boundary conditions in the plane and sets up Floquet port excitations in the positive and negative direction perpendicular to the plane. Thus the values of the the structure parameters are $D=5.6 \mathrm{~mm}, w=3.2 \mathrm{~mm}, h=0.6 \mathrm{~mm}$ are calculated by using Equation (1) and (2). By using some adjustment in the CST simulation the optimized parameters are $D=5.8 \mathrm{~mm}, w=2 \mathrm{~mm}, h=0.6 \mathrm{~mm}$.

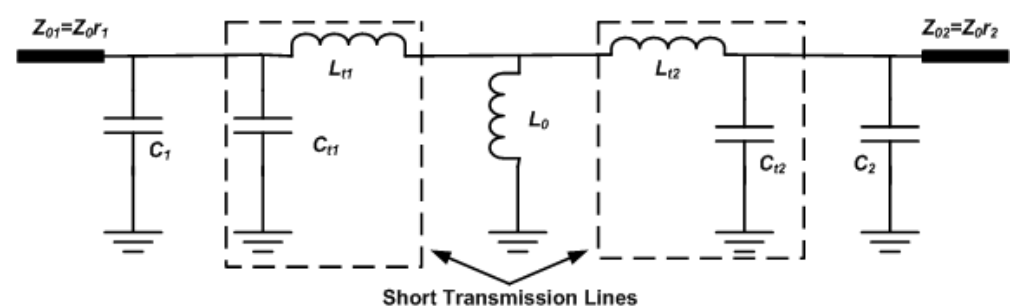

(a)

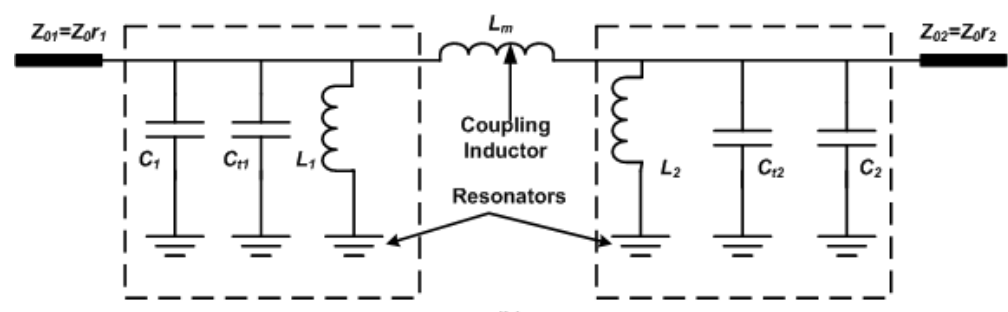

(b)

Figure 3. (a) The equivelent circuit in the form ot T-network, (b) The equivelent circuit in the form of $\pi$-network 


\section{Results and Discussions}

The Scattering parameters of the proposed FSS are shown in the Figure 4 and Figure 5 for different values of incident angles. These figures show the simulated frequency response of the FSS at $X$ band for TE and TM polarizations at various angles of incidence. It can be seen that the bandwidth decrease with the increase in the angle of incidence. The resonant frequency is shifted to higher frequency with the increase in incident angles. But the overall response is the same. The shift of frequency to higher frequency bands is more rapid in TM polarization case in contrast to TE polarization case. These types of FSS can be used for RCS reduction and improvement of antenna gain and directivity. These types of antennas are usually consists of feed antennas [14-15] with integrated FSS act as a partial reflecting surfaces and form resonating cavity and thus increase the gain and directivity of the antennas. In some cases these FSS are used as an artificial magnetic conductor as a ground plane in various antennas to reduce the surface current and increase the bandwidth and reduce the coupling to the near by transmitting antennas. Furthermore, the FSS is used for the radome application. The results show that the FSS act as a pass band spatial filter allowing only the desired band signals and thus reducing the radar cross section of the antenna which is very important in stealth technology.

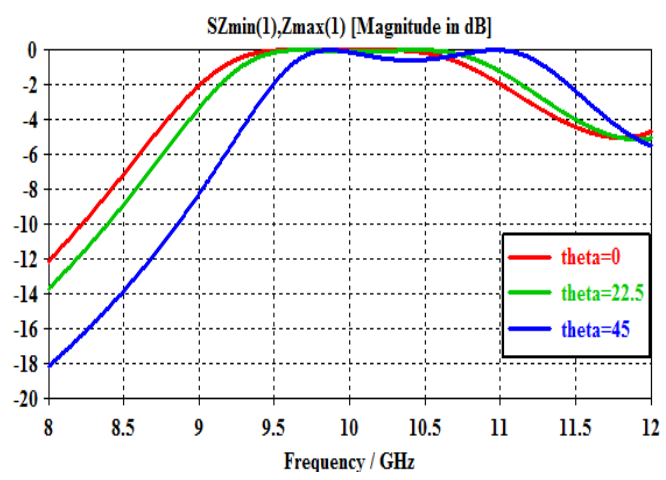

Figure 4. Transmission coeffecint of the $X$ band FSS for TE polarization

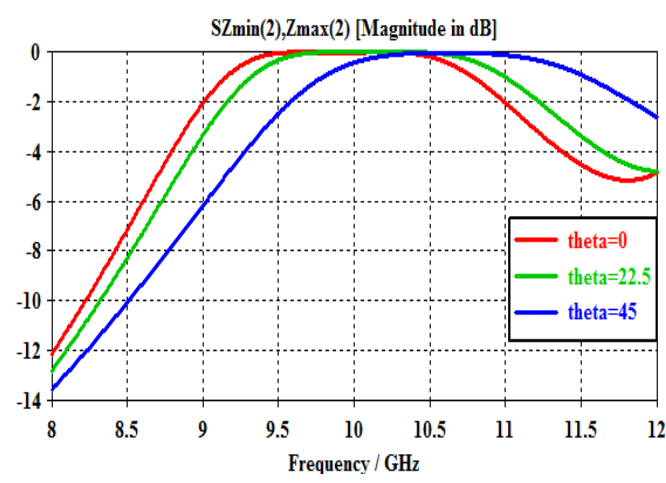

Figure 5. Transmission coeffecint of the $X$ band FSS for TM polarization

\section{Results and Discussions}

A miniaturized FSS is design and analyzed with second-order band pass response at Xband for radome applications. The structure based upon coupled-resonator filter topology using sub wavelength periodic structures composed of capacitive patches and inductive strips. The frequency response for different angles of incidence and polarizations is independence. The structure was analyzed using equivalent circuit approach and design in CST microwave studio. The FSS shows good reflection and transmission characteristics in the design band.

\section{References}

[1] Munk BA. Frequency Selective Surfaces: Theory and Design. New York: Wiley-interscience. 2000.

[2] Bertoni H, Cheo L, Tamir T. Frequency-selective reflection and transmission by a periodic dielectric layer. IEEE Trans. Antennas Propag. 1989; 37(1): 78-83.

[3] Al-Joumayly M, Behdad N. A New Technique for Design of Low-Profile, Second-Order, Band pass Frequency Selective Surfaces. IEEE Trans. Antennas Propag. 2009; 57(2): 452-459.

[4] Kazemzadeh A, Karlsson A. Multilayered wideband absorbers for oblique angle of incidence. IEEE Trans. Antennas Propag. 2010; 58(11): 3637-3646.

[5] Costa F, Monorchio A, Manara G. Analysis and design of ultra thin electromagnetic absorbers comprising resistively loaded high impedance surfaces. IEEE Transa. Antennas Propag. 2010; 58(5): 1551-1558.

[6] Kern D, Werner D. A genetic algorithm approach to the design of ultra-thin electromagnetic bandgap absorbers. Microw. Opt. Technol. Lett. 2003; 38(1): 61-64.

[7] Paquay M, Iriate J, Ederra I, Gonzalo R, Maagt R. Thin AMC structure for radar cross section reduction. IEEE Trans. Antennas Propag. 2007; 55(12): 3630-3638. 
[8] Fu Y, Li Y, Yuan N. Wideband composite AMC surfaces for RCS reduction. Microw. Opt. Technol. Lett. 2011; 53(4): 712-715.

[9] Simms S, Fusco V. Chessboard reflector for RCS reduction. IEEE Electon. Letter. 2008; 44(4): 316317.

[10] Abbaspour A, Sarabandi K, Rebeiz G. Antenna filter-antenna arrays as a class of bandpass frequency-selective surfaces. IEEE Trans. Microw. Theory Tech. 2004; 52: 1781-1789.

[11] Pous R, Pozar D. A frequency-selective surface using aperture-coupled microstrip patches. IEEE Trans. Antennas Propag. 1991; 39(12): 1763-1769.

[12] Sarabandi K, Behdad N. A frequency selective surface with miniaturized elements. IEEE Trans. Antennas Propag. 2007; 55(5): 1239-1245.

[13] Teo PT, Lee K, Lee C. Analysis and design of band-pass frequency-selective surfaces using the FEM CAD tool. International Journal of RF and Microwave Computer-Aided Engineering. 2004; 14(5): 391397.

[14] Ashish S, Kamakshi M, Aneesh, Ansari JA. Slots and Notches Loaded Microstrip Patch Antenna for Wireless Communication. TELKOMNIKA Indonesian Journal of Electrical Engineering. 2015; 13(3): 584-594.

[15] Xue JB, He FJ, Wang D. Analysis and Design of Tag Antenna Based UHF RFID for Libraries. TELKOMNIKA Indonesian Journal of Electrical Engineering. 2015; 13(3): 521-528. 